PROCEEDINGS OF THE

AMERICAN MATHEMATICAL SOCIETY

Volume 137, Number 3, March 2009, Pages 815-824

S 0002-9939(08)09552-X

Article electronically published on September 10, 2008

\title{
REPRESENTATIONS OF THE GENERALIZED KRONECKER QUIVER WITH COUNTABLY MANY ARROWS
}

\author{
NILS MAHRT \\ (Communicated by Birge Huisgen-Zimmermann)
}

\begin{abstract}
Let $Q$ be the generalized Kronecker quiver with countably many arrows and let $k$ be a field. We prove that the category of representations of $Q$ over $k$ has no right almost split morphism whose domain is projective. More precisely, we show that any indecomposable non-projective representation is the image of an epimorphism whose domain has no non-zero projective direct summand. This result does not hold for any finite subquiver of $Q$.
\end{abstract}

\section{INTRODUCTION}

This paper discusses the categories of representations of generalized Kronecker quivers, which are defined as follows.

Definition 1.1. Let $\omega$ be the set of natural numbers and let $\omega^{\prime} \subseteq \omega$. Define the generalized Kronecker quiver $K\left(\omega^{\prime}\right)$ to be the quiver consisting of two vertices $a$ and $b$ and arrows from $a$ to $b$ indexed by $\omega^{\prime}$. The quiver $K\left(\omega^{\prime}\right)$ will be drawn as follows:

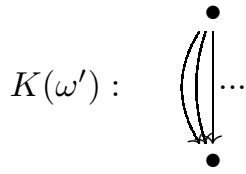

In case $\omega^{\prime}=\omega$, we denote the quiver $K(\omega)$ by $Q$.

Let $k$ be an arbitrary field and $\omega^{\prime} \subseteq \omega$ as above. Recall that a representation of $K\left(\omega^{\prime}\right)$ is given by two vector spaces $V_{a}$ and $V_{b}$ associated to the vertices $a$ and $b$ and by linear transformations from $V_{a}$ to $V_{b}$ associated to the arrows indexed by $\omega^{\prime}$. The category of representations of $K\left(\omega^{\prime}\right)$ over $k$, denoted by $\operatorname{Rep} k K\left(\omega^{\prime}\right)$, is equivalent to the module category of the path algebra $k K\left(\omega^{\prime}\right)$. This category is hereditary, meaning that any subobject of a projective object is again projective (see [Ben98, Theorem 4.1.4).

Following the convention of ASS06, an object is called indecomposable if it is non-zero and does not admit a decomposition as a direct sum of two non-zero subobjects.

Received by the editors November 9, 2006, and, in revised form, May 23, 2007; August 31, 2007; December 20, 2007; and February 18, 2008.

2000 Mathematics Subject Classification. Primary 16G20; Secondary 16G70.

Key words and phrases. Infinite generalized Kronecker quiver, almost split morphism.

(C)2008 American Mathematical Society Reverts to public domain 28 years from publication 
In the last decade, several authors have considered the problem of extending classical results concerning finite quivers to the setting of certain infinite quivers (see e.g. the article [EE05] and its references). In [CDT97, Example 5.3, the path algebra of $Q$ was introduced as the direct limit of path algebras of finite generalized Kronecker quivers (see HU91, p. 182). In D'E00, questions concerning direct products of free and projective representations of infinite generalized Kronecker quivers have been considered. Generalized Kronecker quivers are examples of rooted quivers, i.e. quivers which have no cycles and which do not contain $A_{\infty}$ as a subquiver (see [EOT04] for a characterization). In [EE05, Enochs and Estrada studied when the category of all representations of an infinite quiver admits projective covers. They showed that this is the case if and only if the quiver is rooted and the base ring is left perfect (see EE05, Theorem 3.3).

In the present paper, we prove that for every indecomposable non-projective representation $M$ of $Q$ over $k$ there is a non-split epimorphism $M^{\prime} \longrightarrow M$, where $M^{\prime}$ has no non-zero projective summand (see Theorem 5.1). As a consequence we obtain that all preprojective objects in $\operatorname{Rep} k Q$ are projective (see Corollary [5.3). Moreover, $\operatorname{Rep} k Q$ does not afford any right almost split morphism whose domain is projective (see Corollary 5.5).

The proof of Theorem 5.1 relies on a careful analysis of a projective cover of $M$ and its kernel in case $M$ is not finitely presented. If $M$ is finitely presented, we use that there is a finite subset $\omega^{\prime} \subset \omega$ such that $M$ is in the image of a faithful embedding functor from $\operatorname{Rep} k K\left(\omega^{\prime}\right)$ into $\operatorname{Rep} k Q$ which preserves projective objects (see Lemma 4.2). In particular, this allows us to prove that $M^{\prime}$ above can be chosen to be finitely presented as well.

\section{The KRUll-REMAK-SChMidT PROPERTY}

Let $\omega^{\prime} \subseteq \omega$. In the following we will use the picture

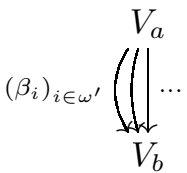

to describe the representation of $K\left(\omega^{\prime}\right)$ which associates to $a$ and $b$ the vector spaces $V_{a}$ and $V_{b}$ and to the arrows in $K\left(\omega^{\prime}\right)$ the linear transformations $\beta_{i}: V_{a} \rightarrow V_{b}$ for $i \in \omega^{\prime}$.

There are only two indecomposable projective objects in the category $\operatorname{Rep} k K\left(\omega^{\prime}\right)$ up to isomorphism. These are represented by the simple projective

$$
P(b)=\underset{k}{\left(\|_{k}^{0}\right.}
$$

and by

$$
P(a)=\quad\left(\operatorname{incl}_{i}\right)_{i \in \omega^{\prime}} \|_{\bigoplus_{i \in \omega^{\prime}} k}^{k}
$$

where incl $_{i}$ denotes the $i$-th inclusion. 
Lemma 2.1. Let $M$ be a finitely generated object in $\operatorname{Rep} k Q$. Then

$$
M \cong M_{1} \oplus \cdots \oplus M_{s},
$$

where all $M_{i}$ are indecomposable. These $M_{i}$ are unique up to isomorphism and permutation.

Proof. To show existence, notice that $M$ can be decomposed as

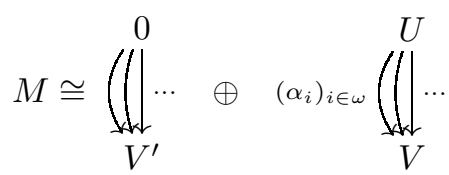

with $\sum_{i \in \omega} \operatorname{im}\left(\alpha_{i}\right)=V$. The vector space $V^{\prime}$ is finite dimensional because $M$ is finitely generated. Therefore the first direct summand of $M$ is isomorphic to a direct sum of finitely many copies of $P(b)$. Hence we can assume without loss of generality that $V^{\prime}=0$. Then

$$
E:=\operatorname{End}_{\text {Rep } k Q}(M) \subseteq \operatorname{End}_{k}(U)
$$

is a finite dimensional algebra because $U$ is finite dimensional. If $E$ has no idempotent except 0 and 1 , then $M$ is indecomposable. If $e \in E$ is an idempotent, the object $M=e M \oplus(1-e) M$ is decomposable. Since the dimension at the upper vertex decreases, this process can be continued and leads to a finite decomposition of $M$.

To show uniqueness it suffices to check that $\operatorname{End}_{\operatorname{Rep} k Q}\left(M_{i}\right)$ is local for all $i$. By the first part of this proof, all $\operatorname{End}_{\operatorname{Rep} k Q}\left(M_{i}\right)$ are finite dimensional. Their only idempotents are 0 and 1 , because otherwise $M_{i}$ would be decomposable. Hence $\operatorname{End}_{\operatorname{Rep} k Q}\left(M_{i}\right)$ is local.

\section{Projective enlargement}

Let $\omega^{\prime \prime} \subseteq \omega^{\prime} \subseteq \omega$ be arbitrary subsets. There is a functor from $\operatorname{Rep} k K\left(\omega^{\prime \prime}\right)$ to $\operatorname{Rep} k K\left(\omega^{\prime}\right)$ which can be defined as a left adjoint to the forgetful functor. This has been done in a more general situation in [EOT04, but the more explicit description given here will be needed to describe the images of these functors in Lemma 4.2 . This lemma will be essential in the proof of the main result, Theorem 5.1, of this paper.

Definition 3.1. Let the projective enlargement be the following covariant functor:

$$
P_{\omega^{\prime \prime} \omega^{\prime}}: \operatorname{Rep} k K\left(\omega^{\prime \prime}\right) \rightarrow \operatorname{Rep} k K\left(\omega^{\prime}\right)
$$

For a representation $\left.\quad \alpha_{i}\right)_{i \in \omega^{\prime \prime}} \underset{V}{U}\left(\|_{\downarrow} \cdots \in \operatorname{Rep} k K\left(\omega^{\prime \prime}\right)\right.$ define

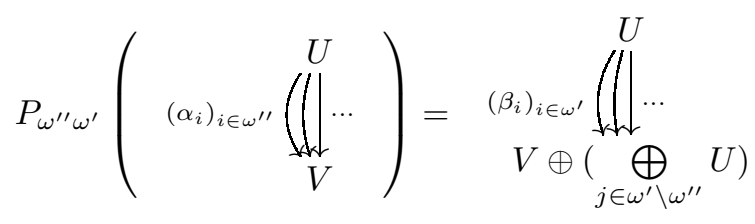


where $\beta_{i}=\left\{\begin{array}{cc}\left(\begin{array}{c}\alpha_{i} \\ 0\end{array}\right) & i \in \omega^{\prime \prime} \\ \left(\begin{array}{c}0 \\ \operatorname{incl}_{i}\end{array}\right) & i \notin \omega^{\prime \prime}\end{array}\right.$

For a morphism $(f, g)=\left(_{i}\right)_{i \in \omega^{\prime \prime}} \underset{V}{\stackrel{U}{\longrightarrow} \underset{g}{\longrightarrow} Y}$

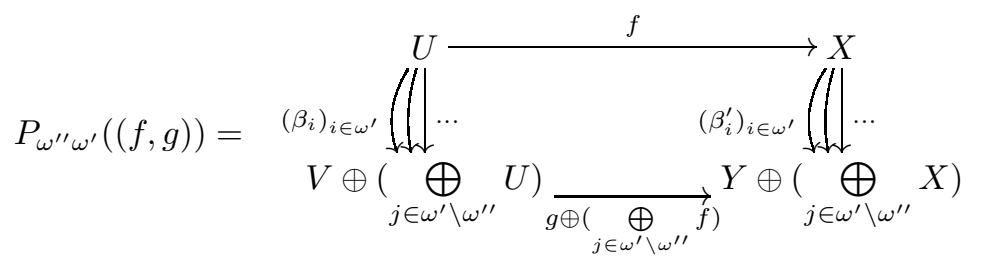

The functor $P_{\omega^{\prime \prime} \omega^{\prime}}$ is called a projective enlargement because of the following result.

Lemma 3.2. The functor $P_{\omega^{\prime \prime} \omega^{\prime}}$ is a faithful embedding of the category $\operatorname{Rep} k K\left(\omega^{\prime \prime}\right)$ into $\operatorname{Rep} k K\left(\omega^{\prime}\right)$, mapping $P(a)$ to $P(a)$ and $P(b)$ to $P(b)$. If the representations $M, N \in \operatorname{Rep} k K\left(\omega^{\prime \prime}\right)$ do not contain $P(b)$ as a direct summand, then

$$
\operatorname{Hom}_{\operatorname{Rep} k K\left(\omega^{\prime \prime}\right)}(M, N) \stackrel{P_{\omega^{\prime \prime} \omega^{\prime}}}{\longrightarrow} \operatorname{Hom}_{\operatorname{Rep} k K\left(\omega^{\prime}\right)}\left(P_{\omega^{\prime \prime} \omega^{\prime}}(M), P_{\omega^{\prime \prime} \omega^{\prime}}(N)\right)
$$

is an isomorphism.

Proof. It is straightforward to check the first part of the lemma. For the second part one uses that a representation $\quad\left(\alpha_{i}\right)_{i \in \omega^{\prime \prime}} \underset{V}{\left(\|_{N}\right.}$ which does not have $P(b)$ as a direct summand satisfies

$$
\sum_{i \in \omega^{\prime \prime}} \operatorname{im}\left(\alpha_{i}\right)=V
$$

The next result is proved by checking the definition.

Lemma 3.3. The forgetful functor is right adjoint to projective enlargement.

Note that the dual construction of projective enlargement yields the left adjoint functor of the forgetful functor.

\section{Finitely PRESENTED OBJECTS}

For finite quivers one often considers finite dimensional representations. For the quiver $Q$ this does not seem to lead to a reasonable simplification as the important objects $P(a)$ and its dual, which is indecomposable injective, are of infinite dimension. Instead one can consider finitely presented objects which lie in the images of the projective enlargement functors $P_{\omega^{\prime} \omega}$ for finite subsets $\omega^{\prime} \subset \omega$ (see Lemma 4.2).

Let $\operatorname{rep} k Q$ denote the full subcategory of finitely presented objects in $\operatorname{Rep} k Q$. This is an abelian category (see [CDT97, Example 5.3 and [Hov01, Lemma 1.6). 
Lemma 4.1. Let $M$ be an indecomposable object in rep $k Q$ which is not isomorphic to $P(b)$. Then $M$ is the cokernel of a monomorphism

$$
\bigoplus_{i=1}^{l} P(b) \longrightarrow \bigoplus_{i=1}^{n} P(a)
$$

for certain integers $l \geq 0$ and $n \geq 1$.

Proof. Consider an indecomposable object $M=\left(\alpha_{i}\right)_{i \in \omega} \underset{V}{\left(\|_{W}\right.}$ in rep $k Q$. Since $M$ is finitely presented and not isomorphic to $P(b)$, it follows that $n=\operatorname{dim}_{k} U$ is finite and at least 1. Because $M$ is indecomposable, the vector space $V$ is covered by the images for the maps $\alpha_{i}, i \in \omega$. Hence there is a surjective map $g: \bigoplus_{i=1}^{n} P(a) \rightarrow M$. It follows that the kernel of $g$ is a direct sum of copies of $P(b)$. Since $M$ is finitely presented, the number of copies of $P(b)$ occurring must be finite.

Lemma 4.2. Let $M$ be an indecomposable object in $\operatorname{rep} k Q$. Then there is a finite subset $\omega^{\prime} \subset \omega$ and an object $M^{\prime} \in \operatorname{rep} k K\left(\omega^{\prime}\right)$ with $P_{\omega^{\prime} \omega}\left(M^{\prime}\right) \cong M$.

Proof. This is obvious if $M \cong P(b)$. Otherwise, by Lemma 4.1, $M$ is isomorphic to

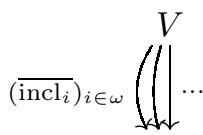

$$
\left(\oplus_{i \in \omega} V\right) / U
$$

for finite dimensional vector spaces $U$ and $V$ where $\overline{\text { incl }_{i}}$ stands for pr $\circ$ incl $_{i}$ and pr denotes the projection $\bigoplus_{i \in \omega} V \rightarrow\left(\bigoplus_{i \in \omega} V\right) / U$. As a finite dimensional subspace, $U$ lies in a finite direct sum $\bigoplus_{i \in \omega^{\prime}} V$. Hence

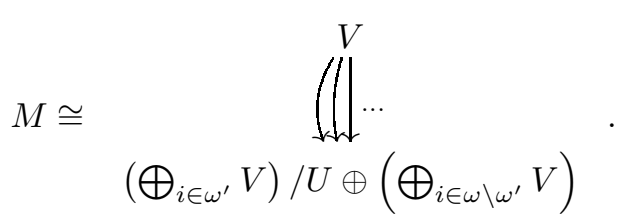

This object is in the image of the functor $P_{\omega^{\prime} \omega}$.

\section{Preprojective objects}

Theorem 5.1. Let $M$ be an indecomposable non-projective representation of $Q$.

Then there is a non-split epimorphism $M^{\prime} \stackrel{f}{\longrightarrow} M$, where $M^{\prime}$ has no projective direct summand except for 0 . If $M$ is finitely presented, $M^{\prime}$ can be chosen finitely presented as well.

Note that Theorem 5.1 does not hold for any finite subquiver of $Q$, which can be seen by the Auslander-Reiten theory.

In the proof of Theorem 5.1, we will make use of the Tits form associated to a finite subquiver of $Q$. For a positive integer $m$, let $K_{m}$ be a subquiver with $m$ arrows. Then the Tits form associated to $K_{m}$ is the quadratic form $q_{K_{m}}: \mathbb{Q}^{2} \rightarrow \mathbb{Q}$ defined by

$$
q_{K_{m}}\left(\begin{array}{l}
u \\
v
\end{array}\right)=u^{2}+v^{2}-m u v .
$$


Let $\left(\begin{array}{c}u \\ v\end{array}\right)$ be the dimension vector of a non-zero object $M$ in $\operatorname{Rep} k K_{m}$. Then by Lemma 2.2 of Rin76, there is the following connection between the Tits form and the dimensions of Hom and $\mathrm{Ext}^{1}$ :

$$
q_{K_{m}}\left(\begin{array}{c}
u \\
v
\end{array}\right)=\operatorname{dim} \operatorname{End}_{\operatorname{Rep} k K_{m}}(M)-\operatorname{dim} \operatorname{Ext}_{\operatorname{Rep} k K_{m}}^{1}(M, M) .
$$

Therefore, if the Tits form does not take a positive value on the dimension vector of $M$, it follows that $\operatorname{Ext}_{\text {Rep } k K_{m}}^{1}(M, M)$ does not vanish, $\operatorname{since}_{\operatorname{dim}_{k}} \operatorname{End}_{\operatorname{Rep} k K_{m}}(M) \geq$ 1.

Proof of Theorem 5.1, Let

$$
M=\quad\left(\alpha_{i}\right)_{i \in \omega} \underset{V}{\frac{U}{V}}
$$

be an indecomposable non-projective representation of $Q$. In particular $U \neq 0$. Denote a projective cover of $M$ by $P(M) \rightarrow M$. The object $P(M)$ is isomorphic to a direct sum of $\operatorname{dim}_{k} U$ copies of $P(a)$. The kernel of the projective cover is isomorphic to a direct sum of copies of $P(b)$. Let $\Omega(M)$ denote this kernel.

The object $M$ is finitely presented if and only if $\Omega(M)$ is finite dimensional over $k$. The "only if" part of this claim was shown in Lemma 4.1. To prove the other direction suppose that $\operatorname{dim}_{k} \Omega(M)=l<\infty$. Hence $M$ is the cokernel of a monomorphism $\bigoplus_{i=1}^{l} P(b) \rightarrow \bigoplus_{I} P(a)$ for some index set $I$. The image of this map is a finite dimensional subspace of $\bigoplus_{I} P(a)$ and therefore has to lie in a finite direct subsum $\bigoplus_{I^{\prime}} P(a)$. Since $M$ does not contain a direct summand isomorphic to $P(a)$, the sets $I$ and $I^{\prime}$ have to be equal. As $I^{\prime}$ is finite this proves that $M$ is finitely presented.

Suppose first that $\Omega(M)$ is infinite dimensional over $k$. One can choose a family of one dimensional submodules $\left(\Omega_{i}(M)\right)_{i \in J}$, such that $\bigoplus_{i \in J} \Omega_{i}(M)=\Omega(M)$. Then for each $i$ there is an induced map $p_{i}$ such that the following diagram commutes:

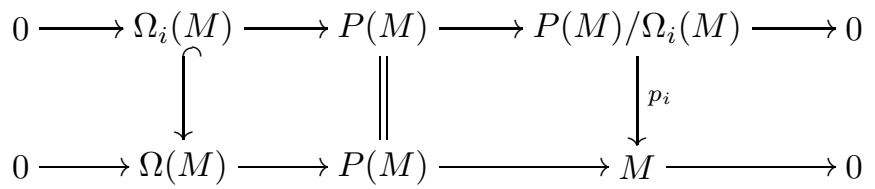

The rows of this diagram are exact. Let $P_{i}$ be the intersection of all submodules $X$ of $P(M)$ such that $X$ is isomorphic to a direct sum of copies of $P(a)$ and contains the image of $\Omega_{i}(M)$ in $P(M)$. This makes $P_{i}$ the smallest submodule with these two properties. Moreover, $P_{i}$ is not only a submodule but also a direct summand of $P(M)$ because both representations are isomorphic to a direct sum of copies of $P(a)$. This yields a commutative diagram with exact rows for each $i$ :

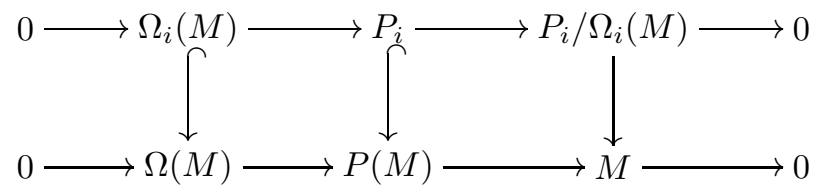


All these diagrams induce a new commutative diagram with exact rows:

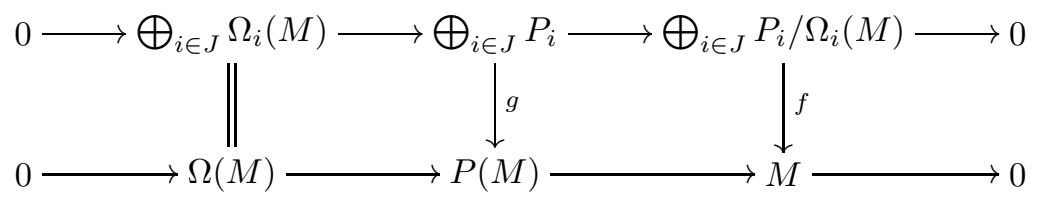

Define $M^{\prime}=\bigoplus_{i \in J} P_{i} / \Omega_{i}(M)$. There is no direct summand of $M^{\prime}$ isomorphic to $P(b)$. Assume that $P$ is a direct summand of $M^{\prime}$ isomorphic to $P(a)$, i.e.

$$
M^{\prime}=\bigoplus_{i \in J} P_{i} / \Omega_{i}(M)=P \oplus M^{\prime \prime} .
$$

Let $v$ be a non-zero element of the one dimensional vector space at the upper vertex of the representation $P$. There is a finite subset $J^{\prime} \subseteq J$ for which $v \in$ $\bigoplus_{i \in J^{\prime}} P_{i} / \Omega_{i}(M)$. This gives an induced map:

$$
\bigoplus_{i \in J^{\prime}} P_{i} / \Omega_{i}(M) \stackrel{h}{\longrightarrow} P
$$

for which $v \in \operatorname{im}(h)$. Thus $h$ is an epimorphism and must split. This means that $P$ is a direct summand of $\bigoplus_{i \in J^{\prime}} P_{i} / \Omega_{i}(M)$. Since $\bigoplus_{i \in J^{\prime}} P_{i} / \Omega_{i}(M)$ is finitely presented, it has a unique decomposition by Lemma 2.1. Hence there exists $i \in J^{\prime}$ such that $P_{i} / \Omega_{i}(M)$ has a direct summand isomorphic to $P(a)$, which contradicts the minimality of $P_{i}$. This means that the assumption was wrong and $M^{\prime}$ has no projective direct summand except 0 .

To finish the proof in case $\Omega(M)$ is infinite dimensional, it remains to check that $f$ is a non-split epimorphism. Since both $\bigoplus_{i \in J} P_{i}$ and $P(M)$ are isomorphic to a direct sum of copies of $P(a)$, it follows that the image of $g$ is a direct summand of $P(M)$, i.e. $P(M)=\operatorname{im}(g) \oplus P^{\prime}$ for some object $P^{\prime}$. Because $\Omega(M) \subseteq \operatorname{im}(g)$, this means that $P^{\prime}$ is a direct summand of $M$. This implies $P^{\prime}=0$ and thus $g$ is an epimorphism. Hence $f$ has to be an epimorphism. Assume $f$ splits. The kernel of $f$ is the kernel of $g$, thus projective. Since $M^{\prime}$ does not contain a proper projective direct summand, it follows that $\operatorname{ker}(g)=\operatorname{ker}(f)=0$. Thus $f$ is an isomorphism. Since $|J|=\operatorname{dim}_{k} \Omega(M)$ is infinite, $M$ is decomposable, contradicting the assumption. Hence $f$ does not split.

Now we consider the case when $\operatorname{dim}_{k}(\Omega(M))=l<\infty$. Since $M$ is indecomposable and finitely presented, it follows by Lemma 4.1 that there is an exact sequence:

$$
0 \longrightarrow \bigoplus_{i=1}^{l} P(b) \longrightarrow \bigoplus_{i=1}^{n} P(a) \longrightarrow M \longrightarrow 0
$$

where $n, l \geq 1$ as $M$ is not projective. By Lemma 4.2 this sequence is in the image of the projective enlargement functor $P_{\omega^{\prime} \omega}$ for a finite subset $\omega^{\prime} \subset \omega$. Let $m$ be the cardinality of $\omega^{\prime}$ and denote $K\left(\omega^{\prime}\right)$ by $K_{m}$ as in the paragraph preceding the proof of Theorem [5.1. Since the quiver $Q$ is infinite, one may assume that

$$
m \geq \frac{n^{2}+l^{2}}{n l}
$$


Let $L$ be an object in $\operatorname{Rep} k K_{m}=\operatorname{Rep} k K\left(\omega^{\prime}\right)$ such that $P_{\omega^{\prime} \omega}(L)=M$. The dimension vector $\operatorname{dim}(L)$ of $L$ satisfies

$$
\operatorname{dim}(L)=\operatorname{dim}\left(\bigoplus_{i=1}^{n} P(a)\right)-\operatorname{dim}(\Omega(L))=\left(\begin{array}{c}
n \\
n m
\end{array}\right)-\left(\begin{array}{l}
0 \\
l
\end{array}\right)=\left(\begin{array}{c}
n \\
n m-l
\end{array}\right) .
$$

Applying the Tits form of $K_{m}$ to $\operatorname{dim}(L)$ yields:

$$
\begin{gathered}
q_{K_{m}}\left(\begin{array}{c}
n \\
n m-l
\end{array}\right)=n^{2}+(n m-l)^{2}-m\left(n^{2} m-n l\right) \\
\quad=n^{2}+n^{2} m^{2}-2 n m l+l^{2}-n^{2} m^{2}+n m l \\
=n^{2}-n m l+l^{2} \leq n^{2}-n \frac{n^{2}+l^{2}}{n l} l+l^{2}=0 .
\end{gathered}
$$

By the paragraph directly preceding the proof of Theorem 5.1, this implies that $\operatorname{Ext}_{k K_{m}}^{1}(L, L) \neq 0$. Hence there is a non-split exact sequence in $\operatorname{Rep} k K_{m}$ :

$$
0 \longrightarrow L \longrightarrow L^{\prime} \longrightarrow L \longrightarrow 0
$$

In particular, $L^{\prime}$ is finitely presented, since it is a finite dimensional representation of the finite quiver $K_{m}$. Applying $P_{\omega^{\prime} \omega}$ to this sequence yields a non-split exact sequence:

$$
0 \longrightarrow M \longrightarrow M^{\prime} \stackrel{f}{\longrightarrow} M \longrightarrow 0
$$

The module $M^{\prime}$ is finitely presented because any finite presentation of $L^{\prime}$ is mapped to a finite presentation of $M^{\prime}$ under $P_{\omega^{\prime} \omega}$ by Lemma 3.2. The object $M^{\prime}$ has no projective direct summand except 0 because there is no non-zero morphism from $M$ into a projective module in the hereditary category $\operatorname{Rep} k K(\omega)$.

Let $C$ denote a subcategory of $\operatorname{Rep} k Q$. Recall that an object $P \in C$ is called splitting projective in $C$ if each surjective morphism $X \longrightarrow P$ with $X$ in $\operatorname{Add} C$ is a splittable surjection. The following definition is motivated by a definition of Auslander and Smalø in [AS80] who considered Artin algebras and finitely generated modules.

Definition 5.2. Let $\mathcal{P}_{0}$ denote the class of splitting projective objects in $C$. For $m \geq 1$ let $\mathcal{P}_{m}$ be the class of splitting projective objects in the full subcategory of $C$ consisting of those objects in $C$ with no direct summand in $\mathcal{P}_{0} \cup \cdots \cup \mathcal{P}_{m-1}$. Then we define $C \cap \operatorname{Add}\left(\bigcup_{m \geq 0} \mathcal{P}_{m}\right)$ to be the class of preprojective objects in $C$.

Corollary 5.3. In $\operatorname{Rep} k Q$ and $\operatorname{rep} k Q$ all preprojective objects as defined above are projective.

Proof. It suffices to show that $\mathcal{P}_{1}=\emptyset$. This follows from Theorem 5.1.

In [ASS06], Chapter IV.1, a right almost split morphism is defined as follows:

Definition 5.4. A morphism $M^{\prime} \stackrel{f}{\longrightarrow} M$ is right almost split if

(1) it is not a split epimorphism and

(2) any morphism $X \longrightarrow M$ which is not a split epimorphism can be factored through $f$.

Corollary 5.5. Neither $\operatorname{Rep} k Q$ nor $\operatorname{rep} k Q$ afford a right almost split morphism $P \longrightarrow M$ with projective $P$. 
Proof. Suppose

$$
P \stackrel{f}{\longrightarrow} M
$$

is a right almost split morphism with $P$ being projective. If $M$ is indecomposable, there is a non-split morphism $M^{\prime} \longrightarrow M$ where $M^{\prime}$ has no projective direct summand by Theorem 5.1. Thus this morphism factors through $f$. Hence there is a non-zero morphism $M^{\prime} \longrightarrow P$ and $M^{\prime}$ must have a projective direct summand.

This means that $M$ is decomposable, i.e. $M=M_{1} \oplus M_{2}$ with $M_{i} \neq 0$. Let incl denote the inclusion of $M_{1}$ into $M$. Then incl factors through $f$ :

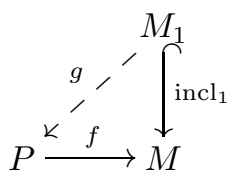

for some $g$. Since incl ${ }_{1}$ is a monomorphism, $g$ is a monomorphism as well. Then $M_{1}$ is projective as a subobject of a projective object in a hereditary category. Analogously $M_{2}$ and hence $M$ is projective. Hence the morphism $f$ splits, which is a contradiction.

Corollaries 5.3 and 5.5 do not hold in the category of representations of any finite subquiver of $Q$. This can be seen by the Auslander-Reiten theory for Artin algebras.

\section{ACKNOWLEDGMENTS}

I thank Claus Michael Ringel for supervising my Diplomarbeit [Mah06], from which this article has been extracted. I further thank Rolf Farnsteiner and the referees for detailed comments and helpful suggestions.

\section{REFERENCES}

[ASS06] Ibrahim Assem, Daniel Simson, and Andrzej Skowroński, Elements of the representation theory of associative algebras. Vol. 1, London Mathematical Society Student Texts, vol. 65, Techniques of representation theory, Cambridge University Press, Cambridge, 2006. MR2197389 (2006j:16020)

[AS80] M. Auslander and Sverre O. Smalø, Preprojective modules over Artin algebras, J. Algebra 66 (1980), no. 1, 61-122. MR591246 (83a:16039)

[Ben98] D. J. Benson, Representations and cohomology. I, second ed., Basic representation theory of finite groups and associative algebras, Cambridge Studies in Advanced Mathematics, vol. 30, Cambridge University Press, Cambridge, 1998. MR.1644252 (99f:20001a)

[CDT97] Riccardo Colpi, Gabriella D'Este, and Alberto Tonolo, Quasi-tilting modules and counter equivalences, J. Algebra 191 (1997), no. 2, 461-494. MR1448804 (98g:16003)

[D'E00] Gabriella D'Este, Free modules obtained by means of infinite direct products, Algebra and its applications (Athens, OH, 1999), Contemp. Math., vol. 259, Amer. Math. Soc., Providence, RI, 2000, pp. 161-173. MR1778499 (2001g:16006)

[EE05] Edgar Enochs and Sergio Estrada, Projective representations of quivers, Comm. Algebra 33 (2005), no. 10, 3467-3478. MR2175445 (2007b:16035)

[EOT04] Edgar Enochs, Luis Oyonarte, and Blas Torrecillas, Flat covers and flat representations of quivers, Comm. Algebra 32 (2004), no. 4, 1319-1338. MR2100360 (2006d:16024)

[HU91] Dieter Happel and Luise Unger, A family of infinite-dimensional non-self-extending bricks for wild hereditary algebras, Representations of finite-dimensional algebras (Tsukuba, 1990), CMS Conf. Proc., vol. 11, Amer. Math. Soc., Providence, RI, 1991, pp. 181-189. MR.1143851 (93c:16014)

[Hov01] Mark Hovey, Classifying subcategories of modules, Trans. Amer. Math. Soc. 353 (2001), no. 8, 3181-3191 (electronic). MR.1828603 (2002i:13007) 
[Mah06] Nils Mahrt, Darstellungen des verallgemeinerten Kronecker-Köchers mit abzählbar vielen Pfeilen, Diplomarbeit, Fakultät für Mathematik, Universität Bielefeld, July 2006.

[Rin76] Claus Michael Ringel, Representations of K-species and bimodules, J. Algebra 41 (1976), no. 2, 269-302. MR0422350 (54:10340)

Fakultät für Mathematik, Universität Bielefeld, Postfach 1001 31, 33501 Bielefeld, Germany

E-mail address: nmahrt@math.uni-bielefeld.de 